\title{
Road surface numerical reconstruction based on image matching
}

\author{
Zhigang $\mathrm{Hu}^{1, a}$, Yunfeng Qiu, \\ ${ }^{1}$ Department of Mechanical Engineering, Wuhan Polytechnic University, China \\ awhhzg@qq.com, b384913874@qq.com
}

Keywords: image matching, road roughness, approach degree, harmonic superposition method, road surface reconstruction.

Abstract. By collecting different grade road images, standard sample library was established. Using fuzzy approach degree algorithm, the approach degree of reconstructed road surface and criterion of road was determined. Then power spectrum of road roughness was built. According to the parameters of power spectrum, we can obtain a $3 \mathrm{~d}$ figure of road surface by improved harmonic superposition method.

\section{Introduction}

Road roughness is the main input of vehicle vibration, and numerical simulation of road spectrum is the key technology of Virtual Proving Ground (VPG)[1].

At present, there are many papers about random road surface reconstruction, but most are used of traditional numerical method. Such as, harmonic superposition method[2], trigonometric series method, white noise filtering method[3], inverse fast fourier transform (IFFT) method that based on power spectral density (PSD)[4], time series of time-domain model and so on[5]. Some techniques and methods, such as image processing and pattern recognition have been applied on breakage and detection of road[6].

This paper proposed digital image processing methods. The approach degree that test pavement and each grade road would be calculated. Preceding calculation results would be used to calculation of test road power spectral parameters of road roughness. The $3 \mathrm{~d}$ surface figure would be obtained with the improved harmonic superposition method.

\section{Standard sample database construction}

In order to establish standard sample database of road surface, we should collect numbers of real images for different grade of the road surface. These images would be the standard sample of grade road surface. This paper from perspective of theoretical basis and preliminary discussion collected four different grades of road surface images. As shown in Fig.1, a simple samples database of road surface has been established. We have collected four real images of road surface samples on different grade roads, and built roads sample database.

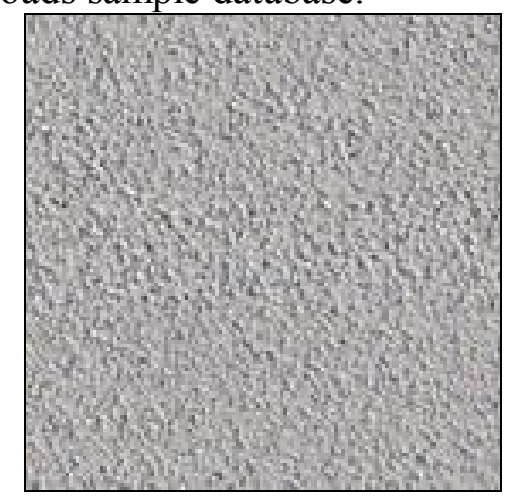

Road surface sample 1

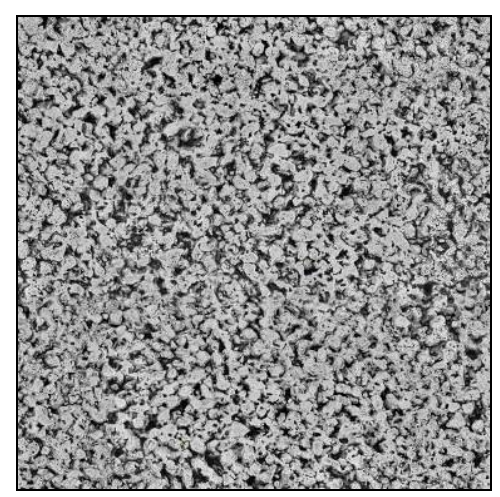

Road surface sample 2 


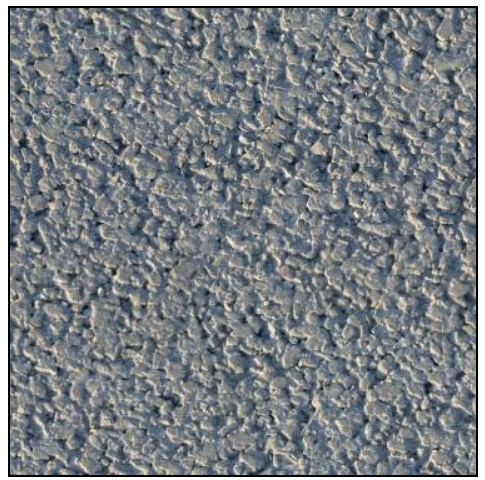

Road surface sample 3

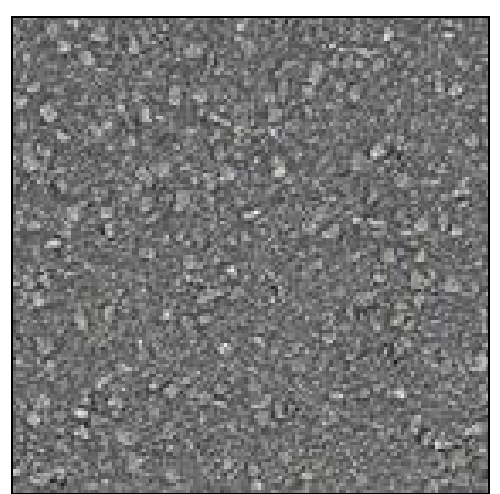

Road surface sample 4

Fig. 1 Road surface samples of different grade road

The feature is extracted to process the images of standard road surface sample. Ordinarily the road surface images collected is colorized. Its color is inessential in the process of road surface grade of recognition. The color of the road surface has a little influence on the results of identification. The color images take much more memory than grayscale images. So we should convert color images to grayscale images.
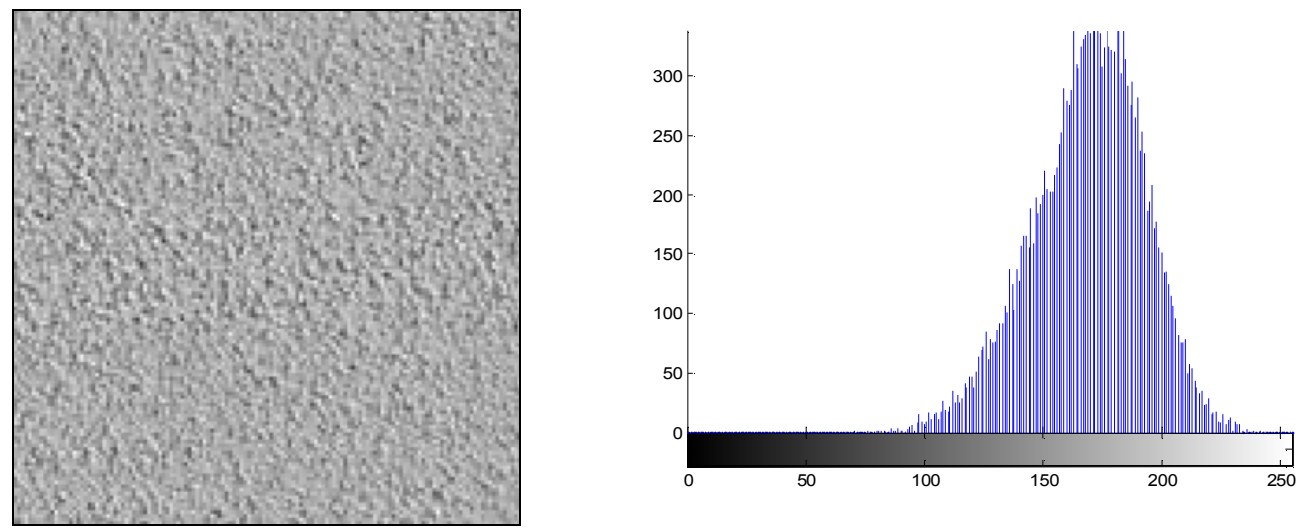

Fig. 2 Grayscale image and gray-level histogram of road surface sample 1
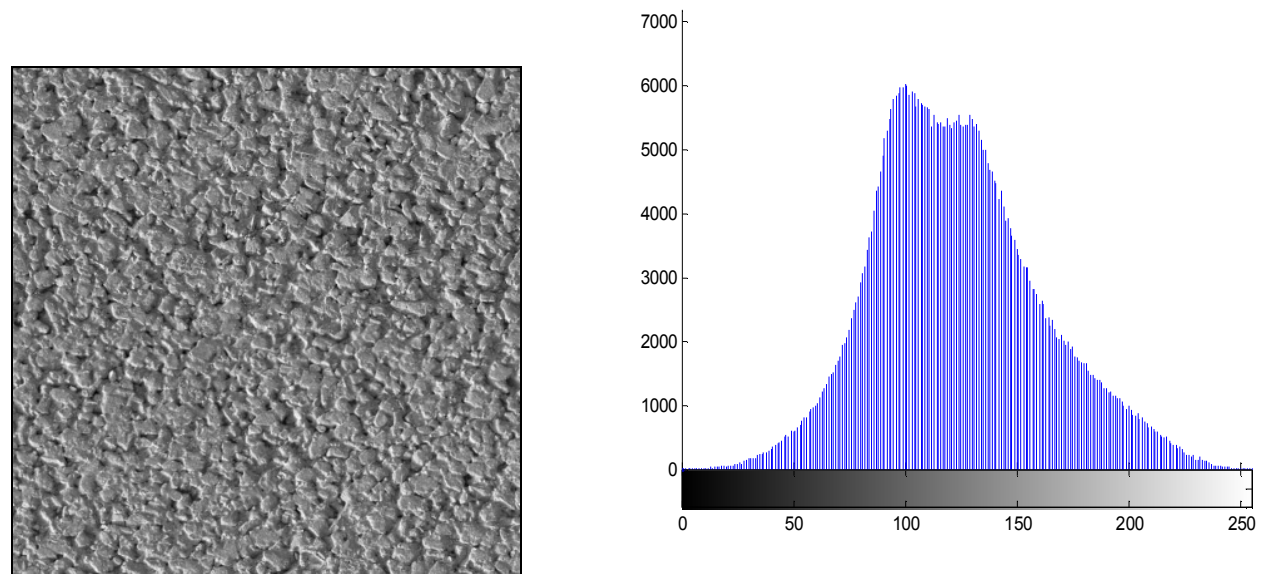

Fig. 3 Grayscale image and gray-level histogram of road surface sample 3

As shown above, Fig. 2 is grayscale image and gray-level histogram of road surface sample 1, Fig. 3 is grayscale image and gray-level histogram of road surface sample 3. Compared with Fig. 3 and Fig. 5, we can get the result that the different gray-level histogram of road surface has a significant difference. So we can take the gray level as the feature of the road pavement. Then, the sample library of standard road would be established. 


\section{Using fuzzy approach degree}

Approach degree can measure close degree of two fuzzy sets[7]. Let $S(\underline{A}, \underline{B})$ is the closeness of fuzzy sets $\underline{A}$ and $\underline{B}$, that $0 \leq S(\underline{A}, \underline{B}) \leq 1$, when $S(\underline{A}, \underline{B})$ is greater, the more close to the two fuzzy sets, and $S(\underline{A}, \underline{B})$ is smaller, the more further to the two fuzzy sets. There are many specific forms of approach degree, such as Hamming close degree, Euclid close degree, maximum and minimum close degree, Grid close degree and so on. Approach degree is mainly used for fuzzy recognition. There are advantages and disadvantages for each close degree on specific application. We should choose an appropriate approach degree according to the actual situation of problem.

We need to find a approach degree that can reflect similar proximity degree between different roads for road recognition. As shown in Eq. 1, we first establish an extremum, and then find the similar approach degree [8].

$$
\min \left\{\left(\sum_{i=1}^{n}\left|\mu_{B}\left(x_{i}\right)-k \mu_{A}\left(x_{i}\right)\right|^{p}\right)^{\frac{1}{p}}, k \in R\right\}
$$

where A, B mean the fuzzy sets, $x_{i}(i=1,2, \cdots, n)$ are the elements of fuzzy set, $\mu_{A, B}\left(x_{i}\right)$ is membership values that $x_{i}$ is affiliated to the collection $\mathrm{A}$ and $\mathrm{B}, k$ is the approach degree value of collection $\mathrm{A}$ and B.

Assuming $p=2, k=\frac{\sum \mu_{A \cup B}\left(x_{i}\right) \mu_{A \cap B}\left(x_{i}\right)}{\sum \mu_{A \cup B}{ }^{2}\left(x_{i}\right)}$ is the approach degree of two fuzzy sets A and B when the Eq. 1 obtain the minimum value. The degree is a blend of two similar characteristics of fuzzy sets. For the two road surface $\mathrm{X}$ and $\mathrm{Y}$, we shall obtain the following equation for approach degree:

$$
\sigma=\frac{\sum \max \left\{X_{i}, Y_{i}\right\} \min \left\{X_{i}, Y_{i}\right\}}{\sum\left(\max \left\{X_{i}, Y_{i}\right\}\right)^{2}}
$$

where $\sigma$ is the approach degree of $\mathrm{X}$ and $\mathrm{Y}, X_{i}, Y_{i}$ are the characteristics of the road $\mathrm{X}$ and $\mathrm{Y}$, there is no dimension when calculate them.

When the fuzzy sets A and B has the elements that completely belong to itself and don't belong to itself, the experiment proved that grid closeness degree is a more fair and objective measurement. In practice, it is often used to represent approach degree of fuzzy sets. The general expression of grid closeness degree is as follows:

$$
N_{g}(A, B)=\frac{1}{2}[(A \circ B)+(1-A \otimes B)]
$$

where $A \circ B=\vee_{i=1}^{n}\left\{A\left(x_{i}\right) \wedge B\left(x_{i}\right)\right\}$ is the inner product of $\mathrm{A}$ and $\mathrm{B}, A \otimes B=\wedge_{i=1}^{n}\left\{A\left(x_{i}\right) \vee B\left(x_{i}\right)\right\}$ is the outer product of A and B. the grid closeness degree in Eq. 3 is defined by provisions. In fact, the grid closeness degree does not meet the reflexivity.

For the pattern recognition problem, the standard type is a fuzzy set on the theory of domain of $X$. When using direct pattern recognition method, such as maximum membership degree principle and threshold principle, to solve the problem, the objects are the elements of theory domain $X$. There is another kind of identification, the objects are also the fuzzy set $X$. This kind of problem can use the following choose-closed principle to identify.

Choose-closed Principle: The standard types $A_{1}, A_{2} \cdots, A_{n} \in F(X), B \in F(X)$, are the objects to identify. $N$ is the approach degree of $F(X)$. If

$$
N\left(A_{i}, B\right)=\max \left\{N\left(A_{k}, B\right) \mid k=1,2, \cdots n\right\}
$$

Then, $B$ and $A_{i}$ was the closest, we should determine that $B$ is belong to the category of $A_{i}$. 
Similarity closeness degree and grid closeness degree can be used to calculate the approach degree between test road surface and standard road surface. By choose-closed principle, we can determine road level of test road.

\section{Calculated parameters of road level}

We take the value of approach degree above as membership degree values of test road. Combining membership degree values and roughness spectrum parameter values of all levels of road surface, we can calculate the parameter values of roughness spectrum of test road.

Assuming that the approach degree values between test road and the road from A to E level are $\sigma_{i},(i=1,2, \cdots, 5), \alpha_{i}, \beta_{i}$ are the parameter values of level $i$ road, so, the parameter values of roughness spectrum of test road is:

$$
\begin{aligned}
& \alpha=\sum_{i=1}^{5} \frac{\sigma_{i}}{\sum_{i=1}^{5} \sigma_{i}} \alpha_{i} \\
& \beta=\sum_{i=1}^{5} \frac{\sigma_{i}}{\sum_{i=1}^{5} \sigma_{i}} \beta_{i}
\end{aligned}
$$

We can take the parameter values of roughness spectrum of test road into the rational function of the road power spectral, then, we could obtain the power spectrum function of test road. Using the power spectrum function, we should reconstruct the roughness of three-dimensional test road.

\section{Three-dimensional road reconstruction}

Take such road image in Hubei province china for example, as shown in Fig. 4. Calculating the fuzzy closeness degree, we can obtain the parameters of the road are: $\alpha=0.1315 \mathrm{~m}^{-1}, \beta=0.0021 \mathrm{~m}$, the road level between $\mathrm{A}$ and $\mathrm{B}$, close to A level.

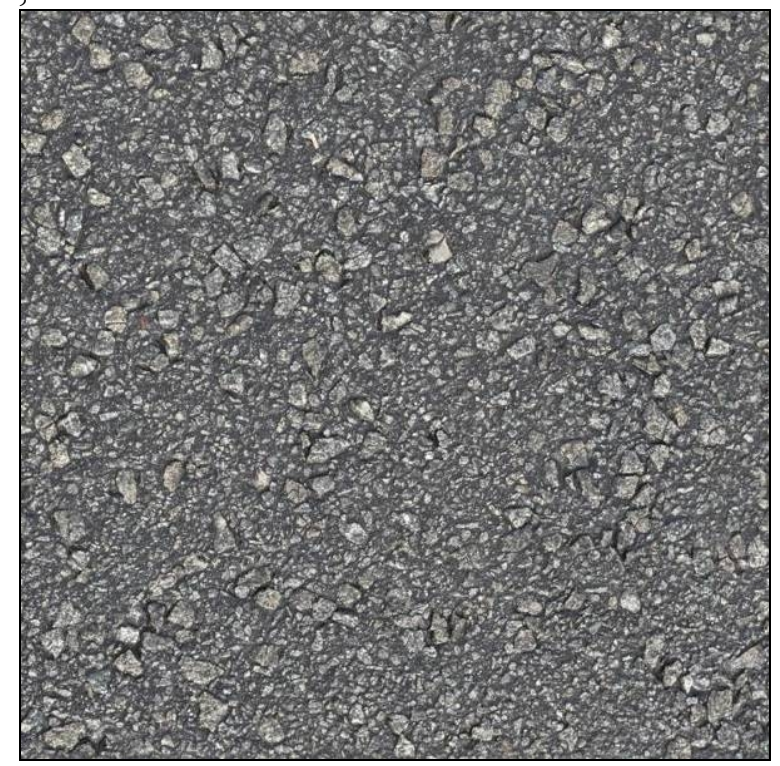

Fig. 4 A image of road surface

Using the algorithm of two-dimensional white noise and extending to $120 \mathrm{~m} \times 120 \mathrm{~m}$. We use matlab/M language to write the program. The simulation frequency range is corresponding to the spatial frequency of the road statistical analysis $\left(0.011 \mathrm{~m}^{-1}<n<2.83 \mathrm{~m}^{-1}\right)$. Spatial sampling interval is $\Delta x=\Delta y=0.3 \mathrm{~m}$. if we reconstruct the sampling road surface, we should get the road surface model which is shown in Fig.5. 


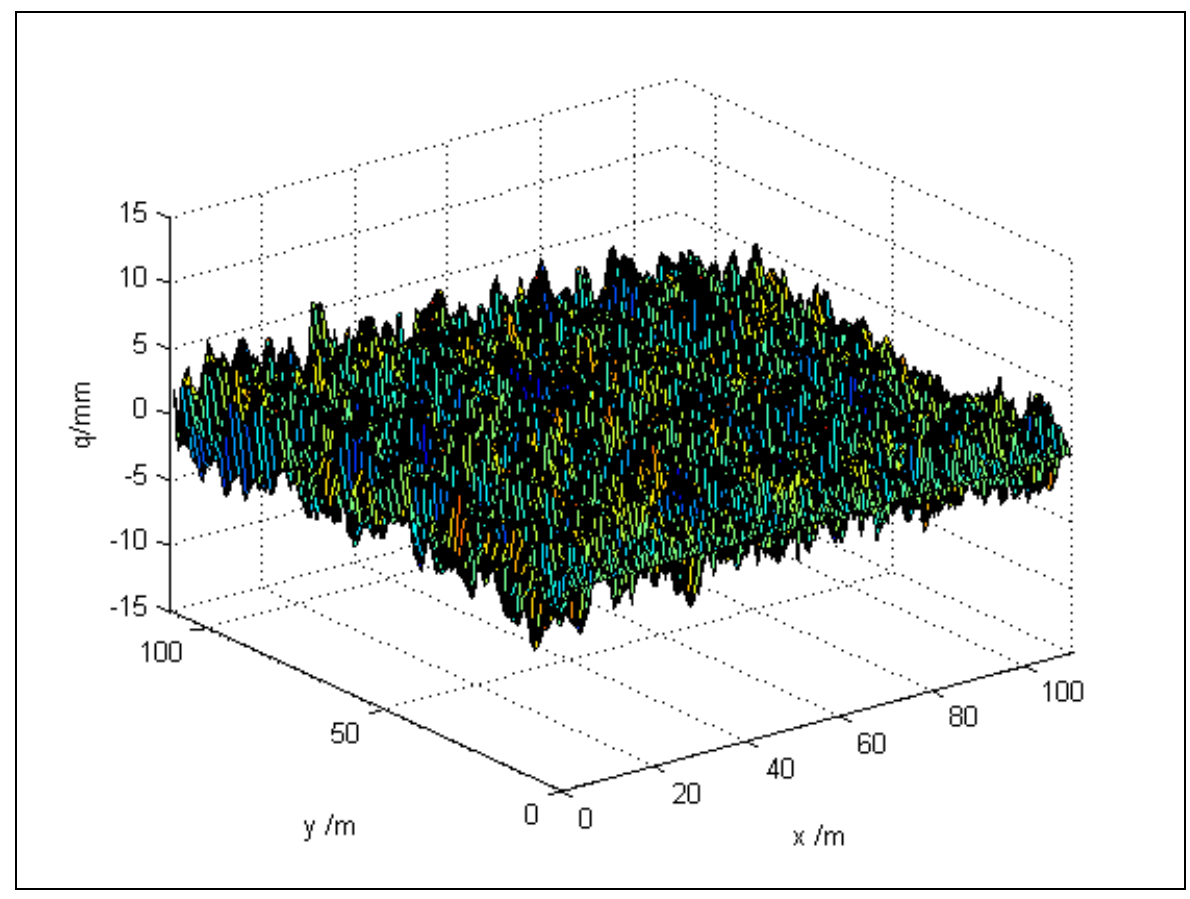

Fig. 5 Reconstruction of 3-dimensional road surface

\section{References}

[1] Z.S.Yu. Theory of automobile. The fifth edition. Beijin: Machinery Industry Press, 2009, 173-174.

[2] K. Bogsjo. Evaluation of stochastic models of parallel road tracks. Probabilistic Engineering Mechanics. 2007, 22: 362-370.

[3] Vincent Rouillard, Michael A. Sek, And Ben Bruscella. Simulation of Road Surface Profiles. Journal of Transportation Engineering, 2001, 5: 247-253.

[4] Y.L.Zhang, Y.F.Zzhong. Time Domain Model of Road Undulation Excitation to Vehicles. Journal of agricultural machinery, 2004,35(2):9-12.

[5] P.B.U.Andersson, W.Kropp. Time domain contact model for tyre/road interaction including nonlinear contact stiffness due to small-scale roughness. Journal of Sound and Vibration, 2008, 318: 296-312.

[6] Z. G. Hu. Research on road surface model system oriented to VPG technique. [PhD thesis]. Wuhan. Huazhong University of Science and Technology, 2011.

[7] Jijian Xie. The method of fuzzy mathematics and its application. the fourth edition. Huazhong University of Science and Technology press. 2013. 4-10.

[8] Tong Xiaojun, Zhang Shemin. Similarity and nearness of fuzzy sets. Proceedings of 2005 International Conference on Machine Learning and Cybernetics, 2005, 8: 2668-2670. 